\title{
IL DEBITO ESTERO IN AMERICA LATINA. ATTORI, CONFLITTI E COALIZIONI
}

\author{
di Fabio Fossati
}

Alcune premesse metodologiche sulla teoria del conflitto

L'obiettivo di questo articolo è l'approfondimento del conflitto distributivo che si è sviluppato sull'applicazione di programmi di aggiustamento ${ }^{1}$ economico da parte dei governi dell'America Latina. Tale conflitto ha coinvolto sia gli attori interni che quelli internazionali, e all'interno di questi ultimi, soprattutto i creditori. La crisi del servizio del debito estero dei paesi dell'America Latina negli anni ottanta, ha rappresentato la variabile condizionale determinante dell'aggiustamento, almeno in questa congiuntura storica. L'analisi comparata di tre paesi chiarirà quanto l'influenza di altri fattori, come il ruolo dei governi o degli attori in gioco, abbiano introdotto elementi di diversità rispetto all'evoluzione storica condizionata dalla variabile internazionale, cioè dal debito estero.

Prima di sviluppare la problematica appena presentata, è necessario riassumere in questo paragrafo la teoria del conflitto, che ho ripreso, e in parte sviluppato, da J. Galtung (1989). Nel-

Questo articolo sviluppa parte della mia tesi di dottorato in Scienza della politica, svolta presso l'Università di Firenze. Intendo ringraziare la CEPAL di Santiago e di Buenos Aires e il CENDES di Caracas per la loro ospitalità, durante il mio soggiorno in America Latina. I miei ringraziamenti vanno, in particolar modo, a $R$. Devlin, $O$. Altimir, $L$. Figeroa, M. Guergill, E. Basualdo, M. Cavarozzi, H. Bejar, J. Iguiniz, V. Fajardo e M. Lopez Maya. Per l'interpretazione (e talvolta per la ricostruzione) degli eventi mi sono servito, oltre che dei testi citati, dello strumento dell'intervista. Ne bo effettuate ottantacinque, a ricercatori, attori governativi, imprenditori e sindacalisti. Per la bibliografia sulla letteratura economica rimando a Fossati (1991). La bibliografia della letteratura secondaria sui tre case studies è limitata per motivi di spazio.

1 Il termine aggiustamento viene spesso usato in alternativa a quello di stabilizzazione: il primo fa riferimento alla bilancia dei pagamenti; il secondo all'inflazione. In Italia comunque viene più usato il termine austerità.

RIVISTA ITALIANA DI SCIENZA POLITICA / a. XXIII, n. 2, agosto 1993 
lo svolgimento della ricerca, ho combinato due approcci complementari: la teoria del potere e la teoria del conflitto. Mentre la prima è ben nota agli studiosi di scienza della politica, e c'è un consenso consolidato sulla definizione di potere, la teoria del conflitto raramente è stata utilizzata in modo sistematico.

La definizione di conflitto di Galtung è la seguente: una relazione tra gruppi sociali o attori politici ${ }^{2}$ i cui scopi sono incompatibili. Nella teoria del conflitto ${ }^{3}$ dunque, il primo passo è rappresentato dalla individuazione degli attori e delle issues di incompatibilità. Il cuore dell'analisi deve poi portare all'identificazione delle due variabili principali: il livello di cristallizzazione e le modalità di risoluzione. Il legame tra queste due variabili è inversamente proporzionale: un conflitto cristallizza quando non viene risolto. Il concetto di cristallizzazione dunque indica la presenza di un conflitto. Tale termine viene utilizzato in quanto permette una differenziazione analitica riguardo all'intensità del conflitto. La cristallizzazione infatti è legata alla durata di un conflitto. Un conflitto a bassa cristallizzazione viene risolto in poco tempo: a volte si esaurisce in una semplice dichiarazione di dissenso, attraverso la quale l'attore manifesta la propria contrarietà su un determinato problema, senza però far seguire a tale dichiarazione comportamenti conflittuali. Invece un conflitto ad alta cristallizzazione richiede molto tempo per essere risolto, o addirittura non viene mai risolto.

Per ciò che riguarda le modalità di risoluzione del conflitto, ne ho individuate dodici, modificando in parte la precedente classificazione di Galtung. Dato che nell'analisi dei casi, tali modalità saranno specificate per ogni singolo attore e durante le diverse fasi storiche, è necessario un sintetico chiarimento del loro significato. La trascendenza si distingue dal compromesso perché i fini degli attori sono realizzati entrambi pienamente. Con il convincimento invece solo un attore realizza il proprio

2 Attribuisco il seguente significato a tali termini: gli attori politici si distinguono dai gruppi sociali per il fatto di essere organizzati. Ad esempio: i salariati sono un gruppo sociale, il sindacato un attore politico; vi sono inoltre gruppi sociali (i lavoratori informali) che non si sono organizzati in attori politici.

${ }^{3}$ Nella teoria del conflitto, viene fatta una distinzione tra livello latente - le issues del conflitto non sono percepite dagli attori - e livello manifesto (le dichiarazioni e i comportamenti). Il conflitto manifesto può essere sia a livello psicologico, che a livello comportamentale (violento o meno). Solo in quest'ultimo caso, il conflitto «esplode»: se esso resta a livello latente o attitudinale, tale situazione verrà identificata come «implosione». 
obiettivo, e l'altro rinuncia volontariamente al suo; tale processo può essere frutto sia di coercizione che di persuasione. Lo scambio implica l'inserimento di un altro fine che permette appunto un sistema di reciproche concessioni. L'integrazione rappresenta il caso in cui c'è una fusione tra due (o più) attori precedentemente in conflitto. La separazione indica la situazione in cui gli attori cessano di interagire. La diversione comporta, come modo per distogliere l'attenzione dal conflitto precedente, l'introduzione (tra gli stessi attori) di un nuovo obiettivo che può essere incompatibile (secondo conflitto) o meno. La multilateralizzazione implica invece l'aggiunta di uno o più attori: nel caso associativo, i vecchi attori si coalizzano contro uno (o più) nuovi; nel caso dissociativo, la coalizione avviene tra attori vecchi e nuovi contro uno (o più) attori vecchi. Tramite la segmentazione un attore riesce a dividere l'altro in due (o più) attori, sviluppando relazioni positive solo con uno dei due. Con la sovversione un attore riesce a provocare un cambiamento di leadership nell'altro attore, sviluppando una relazione positiva con i nuovi leaders. Con la riduzione all'impotenza, un attore elimina o neutralizza l'altro attore. Con l'instaurazione di un rapporto di dominio, un attore impone il proprio obiettivo e non esiste consenso da parte di chi subisce ${ }^{4}$. Le diverse modalità saranno rilevate in tre casi: Argentina, Perú e Venezuela.

\section{Il conflitto sul debito estero e l'aggiustamento economico}

A questo punto è possibile affrontare il tema della risoluzione e della cristallizzazione del conflitto sul debito estero dei paesi dell'America Latina. Come è noto, la crisi del debito si è manifestata nel 1982, quando il Messico non è stato più capace di adempiere ai propri obblighi di pagamento. Il processo di indebitamento presso le banche estere si era sviluppato negli anni settanta, quando l'eccesso di liquidità causato dal flusso

${ }^{4}$ Il conflitto che si risolve in modo asimmetrico (dominio o riduzione all'impotenza) può non essere cristallizzato, dato che questa variabile dipende dai comportamenti. Infine, ho individuato quattro sotto-modalità del dominio: a) repressione. Il dominio è imposto con l'uso della forza; b) frammentazione. La struttura rappresentativa dell'attore è neutralizzata e solo l'exit individuale dei membri è possibile; c) finzione. C'è un accordo ufficiale per risolvere il conflitto, ma l'attore più potente continua ad agire come prima; d) illusione. L'attore meno potente si auto-convince che in futuro il conflitto sarà risolto. 
dei petrodollari era stato indirizzato verso i paesi del terzo mondo, e soprattutto verso l'America Latina. La modalità di risoluzione del conflitto sul mancato pagamento del debito estero è stata, in una prima ipotesi, lo scambio tra riscadenzamento e rifinanziamento ${ }^{5}$ del debito da parte dei creditori e l'applicazione di programmi di aggiustamento economico ${ }^{6}$ da parte dei debitori. Il Fondo Monetario Internazionale (FMI) è stato il garante di tale scambio: banche e governi creditori condizionavano il riscadenzamento del debito all'assenso del FMI sulla politica economica del governo, accompagnato spesso da un prestito del Fondo. Una volta risolta la crisi del 1982, il processo negoziale ha seguito l'approccio case by case, e il servizio del debito non ha più rappresentato un obiettivo incompatibile ${ }^{7}$ tra governi debitori e creditori; semmai il conflitto è cristallizzato con gli attori interni sull'aggiustamento economico. Molti osservatori però, enfatizzando la natura asimmetrica dello scambio, hanno sottolineato che la modalità più rispondente di risoluzione del conflitto sul debito sia in realtà la diversione negativa, che comporta appunto l'aggiunta di un altro fine incompatibile (l'aggiustamento).

Queste considerazioni danno luogo a due generi di conseguenze: l'una riguardante la formulazione dell'ipotesi di ricerca, l'altra la relazione tra le variabili. L'ipotesi della ricerca si basa

5 Il primo termine indica la ridefinizione dei termini di pagamento in un periodo di tempo più lungo, comprendente, a volte, un periodo di grazia, cioè un periodo iniziale in cui il servizio del debito viene sospeso. Il rifinanziamento indica invece l'immissione di crediti freschi al fine di permettere il servizio del vecchio debito.

6 Negli anni ottanta si approfondi una divisione tra le maggiori scuole economiche, che esisteva già da vari decenni, sul tipo di aggiustamento che i governi dell'America Latina dovevano applicare. Gli ideologi liberisti, che si ispiravano al FMI, proposero il modello ortodosso; i neo-strutturalisti, che si ispiravano alla CEPAL, optarono per il modello eterodosso. Il modello ortodosso è basato su politiche monetarie e fiscali restrittive, taglio ai salari, liberalizzazione dei prezzi e del tasso di cambio; il modello eterodosso sul blocco di prezzi, salari e tasso di cambio. Nella fase di aggiustamento strutturale degli anni novanta, le differenze tra le due scuole si sono ridotte. In questa fase sono state applicate la riforma tributaria, le privatizzazioni, l'apertura sia commerciale, che agli investimenti esteri in campo produttivo e finanziario. Sull'argomento, rimando a Fossati (1991).

${ }^{7}$ Nei rari casi in cui alcuni paesi hanno sollevato un conflitto sul servizio del debito, la modalità di risoluzione del conflitto è stata la separazione. Ciò è successo in Perú con Garcia (1985-90), mentre la fase di confronto aperta dall'Argentina di Alfonsin nel 1984 durò poco meno di anno; la moratoria brasiliana del 1987 fu anch'essa di breve durata. Recentemente alcuni governi debitori (Argentina dal 1988 e Brasile dal 1989) avevano dichiarato moratorie tacite di breve/medio periodo, dettate più da difficoltà di bilancia dei pagamenti che da una esplicita strategia di confronto. 
sulla relazione inversa tra conflitto internazionale ed interno, che è conseguenza diretta della diversione. Se infatti il debito estero viene pagato, il conflitto cristallizza tra governo e gli attori interni sui programmi di aggiustamento; se invece il governo dichiara una moratoria, il conflitto cristallizza con i creditori ma non con gli attori interni, non venendo applicato l'aggiustamento. Nelle pagine seguenti, approfondirò la formulazione di tale ipotesi.

Dall'individuazione dello scambio o della diversione (in entrambi i casi con l'aggiustamento) come modalità di risoluzione del conflitto sul debito nasce lo spunto per identificare la relazione tra le due variabili di tipo economico. La maggior parte degli osservatori ha sottolineato che la relazione causale è a due direzioni. Negli anni settanta l'indebitamento è stato la variabile dipendente di politiche economiche che, per evitare l'aggiustamento, hanno fatto ricorso ai crediti esteri al fine di finanziare il deficit fiscale e quello della bilancia dei pagamenti - il primo per permettere gli investimenti pubblici, il secondo quei tassi di cambio che rendevano più economiche le importazioni - o anche per facilitare la fuga di capitali privati. Negli anni ottanta l'aggiustamento è stato la variabile dipendente per permettere il servizio del debito ai creditori ${ }^{8}$.

Le scelte dai governi sul modello di sviluppo hanno portato alla formazione di coalizioni di attori interni favorevoli al protezionismo - basato sul modello dell'industrializzazione per la sostituzione delle importazioni - o al liberismo - che porta all'integrazione con l'economia internazionale. I due modelli di sviluppo, protezionista e liberista hanno portato (in teoria) all'applicazione rispettivamente di politiche economiche espansive e di aggiustamento. Gli attori internazionali (banche e FMI, governi $^{9}$ e imprese $^{10}$ ) sono sempre stati a favore del modello libe-

${ }^{8}$ Il debito estero è stato lo strumento negoziale per convincere coercitivamente i governi dell'America Latina ad applicare l'aggiustamento, ma non ha rappresentato l'unica variabile indipendente. Un'altra è riconducibile al fallimento delle politiche economiche precedenti, sia negli anni sessanta-settanta che negli anni ottanta, quando l'insuccesso dell' aggiustamento ortodosso ed eterodosso ha portato alla necessità dell'inizio della fase dell' aggiustamento strutturale.

9 L'analisi del comportamento di attori statali, come gli Stati Uniti, non è stata formalizzata perché ritenuta non rilevante nella issue in questione; nel testo vi sono comunque riferimenti a casi specifici in cui l'influenza si è verificata. Il ruolo statunitense in America Latina è stato fin troppo enfatizzato, sia da autori realisti che marxisti, che hanno fatto spesso coincidere, a mio avviso a torto, interessi di Stati Uniti e del FMI.

${ }^{10}$ Ho escluso dal modello formale anche le imprese straniere, perché negli anni 
rista: dato che dal bilancio fiscale dello stato derivano sia le sovvenzioni agli attori interni (stipendi degli impiegati pubblici, crediti agli imprenditori, sussidi ai settori poveri della popolazione), che il pagamento del debito estero, essi fanno pressioni sui governi per adottare programmi di aggiustamento e facilitare così il servizio. Indicherò come coalizione protezionista quella formata dai seguenti attori: imprese pubbliche, capitale filoprotezionista, salariati. La coalizione liberista è formata da creditori esteri, grandi gruppi economici, capitale filo-liberista. I grandi gruppi sono quelle imprese che, negli ultimi decenni, hanno diversificato le proprie attività tra industria, agricoltura, commercio, grazie al rafforzamento del loro nucleo finanziario: in principio traggono profitto da qualsiasi situazione, ma storicamente si sono dimostrati molto più favorevoli al modello liberista e all'aggiustamento ${ }^{11}$. Tali gruppi non fanno quasi mai parte delle associazioni imprenditoriali, al contrario degli altri settori del capitale, che si distinguono dai grandi gruppi proprio perché le loro attività sono localizzate e non diversificate. Il capitale filo-liberista raduna i produttori agricoli per l'esportazione, il settore finanziario, gli importatori; il capitale filo-protezionista $\mathrm{i}$ produttori industriali per il mercato interno e per le esportazioni, i piccoli industriali, i produttori agricoli per il mercato interno ${ }^{12}$. Un settimo attore è rappresentato dai cosid-

sessanta e settanta esse erano state in gran parte penalizzate dalle nazionalizzazioni: alcune imprese rimasero, esercitando però un ruolo minore nelle economie dell'America Latina. Tale ruolo era legato a politiche di investimenti tendenti a favorire in alcuni casi il capitale filo-protezionista, ma soprattutto quello filo-liberista (vedi le conclusioni). Nella svolta ho ritenuto opportuno evidenziare solo l'intervento diretto delle imprese straniere, che si ristabilisce solo nell'aggiustamento strutturale degli anni novanta, grazie alle privatizzazioni. Negli anni ottanta, le imprese straniere talvolta sono state contrarie all'aggiustamento perché le loro esportazioni erano danneggiate dal calo della domanda in America Latina. A partire dal lancio della formula dei debt for equity swaps nel 1987, esse hanno iniziato a trarre benefici dalla crisi del debito e hanno aumentato $i$ loro investimenti. Con questa operazione, le imprese straniere compravano (in moneta locale) azioni di imprese (pubbliche o private) in cambio della riduzione del debito pubblico.

"Il modello protezionista infatti è stato accompagnato spesso da politiche economiche espansive che miravano ad una redistribuzione del reddito a favore dei salariati. In ogni caso, in Argentina si è consolidato un polo di gruppi (la cosiddetta patria contratista), che si è formato grazie alle commesse statali, polarizzandosi con l'altro polo, orientato all'esportazione. Il primo polo fa quindi parte della coalizione protezionista: in Argentina dunque vi è una maggiore integrazione tra gruppi e settore industriale. In Venezuela invece la situazione è opposta: scarsa integrazione con lo stato (e con il capitale filo-protezionista), e massima integrazione con il capitale estero. In Perú infine, vi sono relazioni sia con lo stato che con le imprese estere, ma senza un cleavage profondo tra i gruppi.

${ }_{12}$ Alcuni attori imprenditoriali, quali la piccola industria e gli importatori, saranno 
detti esclusi (marginali/informali ${ }^{13}$ urbani e contadini), che in teoria non sono favoriti da nessuno dei due modelli.

Il modello di relazione tra gli attori è quello stellare: tutti gli attori, sia interni che internazionali, si indirizzano al governo per soddisfare i propri interessi. Il governo debitore rappresenta quindi il principale regolatore del conflitto, il boundary role actor tra livello interno e internazionale. Esso, attraverso le politiche economiche, distribuisce benefici e svantaggi ai diversi attori, che saranno suddivisi in tre $^{14}$ categorie: favoriti, penalizzati ed esclusi. Gli attori favoriti ricevono prevalentemente benefici; quelli penalizzati ricevono soprattutto svantaggi; quelli esclusi, che cumulano le condizioni di informalità occupazionale e marginalità abitativa, non sono targets delle politiche economiche (vedi le note 16 e 17). Un altro regolatore del conflitto è il FMI, che svolge questa funzione dopo la crisi del 1982.

L'obiettivo di questo articolo quindi non è solo quello di evidenziare i processi facenti riferimento alla cristallizzazione e alle modalità di risoluzione del conflitto, ma anche di valutare gli effetti di tali eventi sulla struttura di potere tra gli attori. L'effetto dei conflitti sulla struttura di potere sarà determinato dalla comparazione tra le coalizioni favorite e penalizzate nel periodo dell'indebitamento (anni settanta) e in quello della crisi del servizio (anni ottanta). Tale operazione sarà effettuata in due fasi analitiche: prima del paragrafo sui casi, verranno identificate le tendenze comuni; nel paragrafo che approfondirà processi e outcomes dei singoli attori, verranno specificate e spiegate le differenze.

Prima di passare ai case studies, intendo ritornare sull'ipotesi di ricerca. L'individuazione delle due coalizioni infatti porta a modificare l'ipotesi iniziale di relazione inversa tra i conflitti

citati in nota nel paragrafo sugli attori e non saranno analizzati in quello sui casi, ma solo per motivi di spazio.

${ }_{13}$ La marginalità rappresenta l'esclusione dal diritto ad un habitat dignitoso: il marginale è colui che (spesso immigrato) abita nei settori periferici della città, che sono deficitari nei servizi sociali, igienici e nelle condizioni di vita in generale. L'informalità invece fa riferimento all'esclusione dal diritto al lavoro e indica l'assenza di una relazione con lo stato, sotto la forma di flussi economici (sussidi e tributi) e di regolazione legislativa. L'esclusione è definita appunto dalla comunanza, che si verifica spesso, di informalità e marginalità, le cui caratteristiche principali sono la povertà e la scarsa capacità di partecipazione politica.

${ }_{14}$ A livello analitico, non ho ritenuto opportuno formalizzare gli effetti neutrali delle politiche economiche: se tale effetto verrà riscontrato a livello empirico, ciò sarà evidenziato nel paragrafo sugli attori. 
sul debito e sull'aggiustamento. La relazione varrebbe cioè solo per i membri della coalizione protezionista e non per gli attori interni della coalizione liberista (grandi gruppi e capitale localizzato), che è composta anche dai creditori esteri. Con la moratoria dunque il conflitto cristallizzerebbe tra governo e coalizione liberista; il regolare servizio del debito porterebbe invece alla cristallizzazione del conflitto tra governo e coalizione protezionista. Tale argomento sarà approfondito nelle conclusioni alla luce dell'analisi empirica.

\section{L'analisi dei casi}

Per facilitare la comprensione al lettore, anticipo alcune generalizzazioni ${ }^{15}$ che sono il risultato dell'analisi empirica e rappresentano il cuore delle conclusioni dell'articolo. Nel paragrafo precedente ho individuato sette attori principali, la cui dotazione di risorse, a livello di potere potenziale, è diversa. I settori del capitale della coalizione liberista (capitale localizzato, imprese straniere, grandi gruppi) avevano in partenza - prendendo come periodo di riferimento l'inizio di questo secolo - più risorse della coalizione protezionista (capitale localizzato, imprese pubbliche, salariati) e degli esclusi, grazie ad un modello di sviluppo basato sulle risorse naturali. I governi dell'America Latina, fin dagli anni trenta, ma soprattutto a partire dagli anni cinquanta, hanno promosso il modello basato sull'industrializzazione per la sostituzione delle importazioni, penalizzando gli attori con maggiori risorse, quali le imprese straniere (attraverso le nazionalizzazioni), il capitale filo-liberista e i grandi gruppi, che avevano però la possibilità di diversificare le loro attività: vi è stata dunque una diffusione di potere. Durante la fase dell'indebitamento degli anni settanta (e prima), i governi hanno continuato a favorire la coalizione protezionista (imprese pubbli-

15 Sottolineo che tali generalizzazioni valgono solo per $\mathrm{i}$ tre casi in questione, anche se l'evoluzione storica riportata sarebbe ribadita dall'analisi empirica di molti altri paesi dell'America Latina. L'eccezione più rilevante è rappresentata dal caso cileno, dove la fase di aggiustamento iniziata negli anni settanta non solo non è stata interrotta dalla transizione democratica degli anni ottanta, ma addirittura ha comportato la contemporanea applicazione dell'aggiustamento strutturale. Vi è stata inoltre una forte cesura tra politiche ultra-liberiste (fallite) a cavallo tra gli anni settanta e ottanta e politiche (riuscite) nella seconda metà degli anni ottanta, miranti a valorizzare le risorse produttive. La transizione degli anni novanta dunque non ha portato a nessun cambiamento rilevante di politica economica. 
che, capitale localizzato e salariati). Negli esperimenti neo-liberali $^{16}$ a cavallo tra gli anni settanta e ottanta, i governi hanno avvantaggiato la coalizione liberista (grandi gruppi e capitale localizzato) e le imprese pubbliche, ma non ci sono state privatizzazioni, almeno nei casi sotto analisi. La transizione democratica e la crisi del debito hanno cambiato gli equilibri: i governi hanno tentato di raggiungere un compromesso tra gli attori. Nella fase di aggiustamento degli anni ottanta il capitale filo-protezionista è rientrato in gioco, e solo i salariati sono stati penalizzati. Nella fase di aggiustamento strutturale degli anni novanta, anche il capitale filo-protezionista e le imprese pubbliche sono stati penalizzati ${ }^{17}$ e la coalizione liberista è stata di nuovo favorita; con le privatizzazioni sono rientrate in gioco le imprese straniere. La diffusione di potere verso la coalizione protezionista si è arrestata e il processo di conversione del potere potenziale in attuale, da parte della coalizione liberista, si è stabilizzato.

Il rapporto tra grandi gruppi e creditori merita un approfondimento. I gruppi, inclusa la patria contratista argentina (vedi nota 11), sono stati favoriti dai creditori negli anni settanta con crediti diretti, mentre i creditori hanno ricevuto la compensazione (della crisi del servizio) con depositi nei loro conti, attraverso la fuga di capitali (scambio). Inoltre negli anni ottanta, i governi hanno convertito il debito privato (Argentina) o concesso il tasso di cambio privilegiato per il suo servizio (Venezuela): l'alleanza ha resistito. In Perú, debito privato e fuga di capitali, seppur legati, sono stati di minore entità: il debito privato non è stato statizzato. L'alleanza era dunque meno forte $\mathrm{e}$ nel periodo di Garcia c'è stata una tacita rottura. Nelle conclusioni ritornerò su questo argomento.

\footnotetext{
16 Negli anni cinquanta e sessanta i settori marginali erano stati favoriti dallo stato, che aveva consolidato parte degli insediamenti degli immigrati (ad eccezione del Perú); mentre l'alta occupazione era uno strumento che preveniva l'informalità. In realtà, il populismo macro-economico ha provocato la crisi economica e quindi la caduta di quei governi; tali interventi finirono dunque per rappresentare una finzione di risoluzione del conflitto. L'esclusione dei marginali è stata rafforzata nella fase neo-liberista alla fine degli anni settanta, quando l'intervento dello stato si è definitivamente interrotto.

${ }_{17}$ In questo periodo c'è stato un consolidamento dell'esclusione urbana, con la flessibilizzazione del mercato del lavoro (aumento dell'informalità). Con la democrazia, i timidi tentativi di politica sociale, lanciati da Alfonsin e Garcia, non sono riusciti. Nella fase di aggiustamento strutturale, $i$ governi hanno adottato politiche sociali basate sulla distribuzione gratuita del cibo ai più poveri, che sostituisce quella populista basata sui sussidi ai generi di prima necessità che favoriva tutta la popolazione. I marginali sperano (specialmente in Perú) che il liberismo risolva il conflitto nel lungo periodo: in futuro si potrà valutare se si tratti di illusione.
} 
Ecco ora l'analisi dei tre paesi ${ }^{18}$ : Perú (dal 1965), Argentina e Venezuela (dal 1973). Le date fanno riferimento all'inizio della fase dell'indebitamento estero ${ }^{19}$.

In Argentina, il colpo di stato militare del 1976 sancisce il crollo della coalizione protezionista, favorita dalle politiche espansive di Perón. Il tentativo di penalizzazione dei salariati con il Rodrigazo dell'agosto del 1975 era fallito perché il sindacato peronista aveva cristallizzato il conflitto attraverso la proclamazione dello sciopero generale: il taglio ai salari fu revocato (convincimento del governo). Di fronte alla profonda crisi economica e sociale, tutti i settori del capitale, compreso il capitale filo-protezionista, si alleano contro il governo di Isabelita (riduzione all'impotenza). I grandi gruppi e il capitale filo-liberista intendevano reagire al progetto di trasformazione della società, che passava attraverso la riforma del rapporto tra capitale e lavoro, ma anche alle politiche economiche sfavorevoli. Il settore finanziario subì le nazionalizzazioni (dominio), mentre i produttori pampeani furono compensati con la mancata implementazione dell'avverso progetto di imposta sulle terre.

Il governo di Martinez de Hoz favorisce la coalizione liberista e soprattutto il settore finanziario, grazie alla liberalizzazione del tasso di interesse nel 1977. A partire dal 1978, la sopravvalutazione penalizza le esportazioni dei produttori pampeani: il conflitto non cristallizza per motivi di affinità ideologica tra governo e produttori; la revoca delle tasse alle esportazioni, applicata già dal 1976, servì inoltre come strumento di scambio. La sopravvalutazione comunque sancisce la rottura con il FMI (separazione). Il capitale filo-protezionista viene penalizzato dall'apertura commerciale e dal calo dei sussidi alle esportazioni

${ }^{18}$ La scelta dei tre casi segue il criterio dell'eterogeneità rispetto a variabili ritenute chiave (metodo delle differenze). Il Perú è stato scelto perché è l'unico ad avere adottato una strategia conflittuale di lungo periodo con i creditori internazionali. L'Argentina e il Venezuela perché da un lato hanno avuto un debito privato alto (con modalità diverse di risoluzione del conflitto), dall'altro perché in entrambi i paesi vi sono forti sindacati (con una diversa cultura del conflitto). Inoltre nei tre paesi si sono verificati (nel 1989/90) degli estallidos sociales, legati alla protesta del settore marginale/informale della popolazione con diversi gradi di intensità e ampiezza. In tutti i paesi infine c'è stata un'alternanza al potere tra i due partiti (o i due poli nel caso del Perú) principali: ciò permette di valutare l'influenza della variabile politica. Dal paragrafo sugli attori emergerà che in molti casi ho utilizzato il metodo della concordanza, tra variabili indipendenti (comuni) e stesso esito del conflitto.

${ }^{19}$ La ricostruzione degli eventi è stata fatta sulle seguenti fonti: Latin American Weekly (and Regional) Reports (Lettres, Londra), Bimestre Politico-Economico (CISEA, Buenos Aires), Resumen Semanal (DESCO, Lima), Cuadernos (CENDES, Caracas). 
industriali; anche i salariati sono fortemente penalizzati. In nessuno dei due casi il conflitto cristallizza, per un evidente squilibrio nella dotazione di risorse coercitive (dominio). L'economia entra in crisi: dato che non ci sono privatizzazioni rilevanti, imperversa la speculazione.

Nel 1981, con la fine del governo di Martinez de $\mathrm{Hoz}$ viene decisa la svalutazione. Si aprono due anni che portano alla guerra delle Malvinas e alla crisi del debito, ma gli accordi con il FMI non sono attuati. I vari governi prendono decisioni di compromesso, che riguardano (in negativo) i creditori - vengono accumulati arretrati sul servizio degli interessi - (in positivo) il capitale filo-liberista, grazie alla svalutazione, e il capitale filoprotezionista - l'apertura commerciale rientra (Acuña 1990). I grandi gruppi vengono favoriti dall'assunzione della maggior parte del debito privato da parte dello stato, dopo un faticoso processo di negoziazione (convincimento del governo), e dalla promozione industriale che rafforza il polo contratista dei gruppi. A partire dal 1983 aumentano anche i salari e c'è uno scambio, denunciato poi da Alfonsin, tra la reintegrazione dei leader sindacali peronisti nell'arena politica e l'appoggio di tali leader all'amnistia verso i militari, in caso di vittoria peronista. In ogni caso, i conflitti non cristallizzano oppure vengono risolti a breve scadenza: il cambiamento di politiche è parziale e la definizione delle alleanze è rinviata.

Alfonsin, con il governo Grinspun, favorisce la coalizione protezionista: tale cambiamento era emerso da un programma definito in campagna elettorale. Grinspun dichiara una moratoria sul capitale e il conflitto con le banche creditrici cristallizza sull'opportunità di passare attraverso il FMI per riscadenzare il debito: il tentativo comunque non riesce e il governo cede già nel settembre del 1984 . I produttori pampeani sono penalizzati dalla contemporanea presenza di sopravvalutazione e tasse alle esportazioni: il conflitto cristallizza. L'opposizione del blocco di creditori, capitale filo-liberista e grandi gruppi - anche le esportazioni industriali sono penalizzate - costringe Grinspun alle dimissioni nel marzo del 1985 (riduzione all'impotenza).

Il cambiamento del governo Sourrouille è provocato quindi su pressioni di attori sia interni che internazionali. Nella fase del Plan Austral (1985-87), il FMI viene convinto ad appoggiare elementi eterodossi come il blocco di prezzi, salari e tasso di cambio. Vengono favoriti i creditori e i vari settori del capitale, compreso quello filo-liberista - le tasse alle esportazioni calano, 
il progetto di imposta sulle terre non passa (Nun e Lattuada 1991). Il sindacato si oppone con successo al calo dei salari (compromesso): il conflitto cristallizza e durante la presidenza Alfonsin vengono proclamati 13 scioperi generali (Gaudio e Thompson 1990). Dal 1987, Sourrouille tenta di penalizzare il capitale filo-protezionista con l'apertura commerciale e i grandi gruppi del polo contratista con alcune misure ad hoc (Ostiguy 1989). In entrambi i casi il risultato finale è il compromesso.

Nel 1988 con il Plan Primavera, Sourrouille decide di nuovo un cambiamento, dettato dalle reazioni degli attori interni (salariati, capitale filo-protezionista e grandi gruppi): penalizza i creditori con la moratoria tacita anche sugli interessi (separazione), il capitale filo-liberista con il differenziale cambiario contro le esportazioni dei produttori pampeani (dominio); infine sceglie come regolatore internazionale la Banca Mondiale, rinunciando al FMI (segmentazione), grazie all'appoggio degli Stati Uniti (Machinea e Sommer 1990). Creditori ed esportatori cristallizzano il conflitto e reagiscono con la speculazione cambiaria - il dollaro si apprezza, grazie alla vendita continua di $A u$ strales - e il rinvio delle vendite dei beni sul mercato internazionale - impedendo alla banca centrale di incassare valuta. Nel febbraio del 1989 Sourrouille dà le dimissioni (riduzione all'impotenza): era cambiato il ministro del Tesoro degli Stati Uniti e anche la Banca Mondiale aveva abbandonato il governo. I successivi governi sanciscono la fase dell'iper-inflazione, in cui non esistono più alleanze e ogni attore tenta di «salvare la pelle». Non vi riusciranno i salariati perché, di fronte ad un calo dei salari mai registrato fino ad allora, il candidato peronista $\mathrm{Me}$ nem impone a Ubaldini, leader sindacale peronista, una sorta di tregua elettorale. Nel maggio si verificano i saqueos in diverse città argentine e nel giugno anche Alfonsin dà le dimissioni (riduzione all'impotenza).

Con Menem il cambiamento è riconducibile ancora una volta alla crisi, con un programma che emerge solo dopo le elezioni. Il governo Rapanelli penalizza la coalizione protezionista, incluso il polo contratista dei grandi gruppi, con tagli alla promozione industriale. La coalizione liberista non viene premiata a pieno: Rapanelli favorisce il capitale filo-liberista, ma punisce i creditori, con la continuazione della moratoria, che viene comunque appoggiata dal FMI. Entrambi i conflitti cristallizzano: i gruppi reagiscono con l'inflazione, i creditori con la speculazione cambiaria. Rapanelli dà le dimissioni alla fine del 1989. 
Gli subentra Gonzales, con cui non avviene un vero cambiamento. Il conflitto tra grandi gruppi e creditori può essere, in parte, risolto dalla formula debt for privatizations swaps (scambio), in seguito alla quale le imprese pubbliche vengono vendute attraverso l'acquisto del loro debito estero. I gruppi vengono colpiti anche se le misure (la conferma dei tagli alla promozione industriale e il rinvio dei pagamenti dei contratti), sono di compromesso. Gonzales attua anche un piano di conversione di depositi a breve termine in titoli pubblici (Piano Bonex), che penalizza la patria financiera, quindi un settore del capitale filo-liberista: alla fine prevale un compromesso che colpisce solo le banche medio/piccole. Il cambiamento avviene nel giugno del 1990, soprattutto su pressioni dei creditori: il FMI si fa garante di un accordo di compromesso sulla base del quale viene ripreso il servizio mensile (parziale) del debito. Il governo di Gonzales corre sul filo del rasoio: stavolta i gruppi del polo industriale sono colpiti dalla sopravvalutazione del tasso di cambio e dalla caduta dei sussidi alle esportazioni industriali. Gli esportatori agricoli (e agro-industriali) avevano ricevuto lo scambio tra caduta delle tasse e sopravvalutazione. Questi due conflitti cristallizzano portando di nuovo all'inflazione e alla speculazione cambiaria. Gonzales abbandona nel febbraio del 1991; nel frattempo il FMI aveva rotto l'alleanza.

Cavallo ricostruisce l'allenza con il FMI, premiando il capitale filo-liberista - le privatizzazioni sono attuate anche in campo finanziario - e i grandi gruppi - che subiscono però le pressioni sulla riforma impositiva. Cavallo inoltre raggiunge un altro compromesso sul pagamento degli arretrati con i creditori, firmando nell'aprile del 1992 un accordo con le banche nel contesto del piano Brady. Menem ha risolto il conflitto con il capitale filo-liberista (i produttori pampeani) con lo scambio alternato (tra tasse alle esportazioni e sopravvalutazione). Il capitale filo-protezionista invece è penalizzato dall'apertura commerciale: il conflitto cristallizza, ma il dominio non viene impedito; inoltre continua la sopravvalutazione che però colpisce maggiormente i grandi gruppi del settore industriale - alla fine del 1992 vengono reintrodotti alcuni sussidi alle esportazioni industriali, ma si tratta di misure di compromesso. E quasi certo che tutte le imprese pubbliche verranno privatizzate. In questo periodo le perdite salariali sono limitate, ma non c'è un recupero rispetto al 1989; i salariati sono colpiti dalla crescente disoccupazione e dalla flessibilizzazione del mercato del lavoro. La 
strategia di risoluzione del conflitto è la segmentazione: Menem isola Ubaldini e il conflitto non cristallizza con nessuna delle due ali del sindacato peronista. Nel marzo del 1992 il sindacato si riunifica e nel novembre viene proclamato il primo sciopero generale contro Menem. Più che una tardiva cristallizzazione del conflitto, sembra che alcuni leaders sindacali vogliano opporsi al progetto governativo di introduzione del pluralismo sindacale che limiterebbe il loro potere ormai storicamente consolidato.

In Perú, Belaunde (1963-68) non riesce a promuovere una coalizione dominante, e si verifica una crisi da egemonia. In seguito il periodo protezionista di Velasco (1968-75), e quello liberista di Morales Bermudez e Belaunde (1976-82) portano al predominio (di breve durata) di una coalizione sull'altra. In ogni caso, l'aggiustamento è efficace, e l'accordo con il FMI mantenuto, solo durante il governo di Silva Ruete del 1978/79. I salariati sono premiati dai primi due presidenti, con parziali eccezioni nel 1967 e dal 1973, quando viene ottenuto il convincimento dei sindacati, per motivi di vicinanza ideologica, al parziale calo dei salari. Il calo è maggiore con Morales Bermudez e il conflitto cristallizza, ma i cinque scioperi generali non riescono ad evitare la relazione di dominio. Il capitale filo-protezionista viene favorito dalla politica economica di Velasco. Il conflitto cristallizza sulle relazioni tra capitale e lavoro e sul progetto di trasformazione della società: tutto il capitale (compresi naturalmente i grandi gruppi) si mobilita e il golpe riduce Velasco all'impotenza. Morales Bermudez opera una segmentazione ai danni del settore industriale, favorendo gli esportatori e penalizzando i produttori per il mercato interno con l'apertura commerciale: il conflitto cristallizza. Belaunde invece penalizza, almeno in una prima fase, entrambi i settori, che comunque sono compensati dall'effetto espansivo della politica creditizia (scambio). Il capitale filo-liberista subisce il dominio da parte di Velasco a causa delle nazionalizzazioni nei settori minerario, peschiero e finanziario; i grandi produttori agrari vengono ridotti all'impotenza attraverso la riforma agraria, che porta alla costituzione delle cooperative. Il settore agricolo moderno della costa non scompare, ma subirà l'effetto negativo dalla politica creditizia restrittiva, in modo selettivo da parte di Morales Bermudez - attraverso la segmentazione a favore delle cooperative più produttive - in modo generalizzato da parte di Belaunde - che effettua uno scambio con delle compensazioni settoriali. Il settore agricolo tradizionale 
della sierra continua a subire il dominio anche dopo la riforma agraria di Velasco, attraverso la politica agraria sfavorevole (scarsi crediti, bassi prezzi, importazioni a buon mercato). Le conquiste della riforma sono in parte smantellate da Belaunde con la parcellizzazione della cooperative: le strutture rappresentative dei contadini - i gremios, burocrazie controllate spesso dai partiti della sinistra - si oppongono, ma non le comunità di base che non avevano condiviso la scelta delle cooperative.

Con la crisi del debito, Belaunde opta per l'immobilismo: non viene presa nessuna decisione chiara di alleanze; ogni possibile cambiamento, la cui provenienza è internazionale (i creditori), viene attenuato a livello interno. La tendenza è il compromesso: moratoria parziale con i creditori - un accordo con il FMI non viene attuato - e mancata statizzazione del debito privato - la sinistra si era opposta all'approvazione da parte del parlamento di misure simili a quelle di Argentina e Venezuela -, ritiro delle politiche (apertura commerciale e calo dei sussidi alle esportazioni) dannose agli industriali - ma i crediti vengono tagliati (scambio) - mancata attuazione del piano di privatizzazioni. I salariati, che prima avevano ricevuto misure di compromesso, sono penalizzati in misura maggiore; in questa seconda fase il conflitto cristallizza, ma l'intensità degli scioperi generali è bassa. L'effetto di tutto ciò è la crisi economica.

Con Garcia il cambiamento, che è il risultato della crisi del precedente governo, si verifica sia a livello internazionale che interno ed è coerente con il programma della campagna elettorale. Garcia torna a favorire la coalizione protezionista, e i creditori sono penalizzati dalla moratoria (separazione): viene pagato solo il $10 \%$ del reddito da esportazioni e sono rotte le relazioni con il FMI. In teoria anche il capitale filo-liberista è penalizzato, ma come succede in caso di politiche protezioniste, il conflitto è risolto con lo scambio (attraverso i crediti). I grandi gruppi spostano le proprie risorse nell'industria, attraverso i negoziati che Garcia effettua con i rappresentanti dei maggiori gruppi, i cosiddetti 12 apostoles (Durand 1987). Quindi, non c'è opposizione dei gruppi alla moratoria, anche se in principio la retorica liberale li avrebbe portati a opporsi a tali misure. Garcia tenta anche di favorire il settore degli esclusi: i contadini con crediti privilegiati e un sistema di prezzi di garanzia; i marginali urbani con sussidi e programmi di occupazione di emergenza. Ma le risorse sono limitate e solo una piccola percentuale di informali viene favorita: si tratta della ripetizione della vecchia finzione populista (Ballon 1990). 
A partire dal 1987 la politica di alleanze di Garcia fallisce sia con i grandi gruppi (e il capitale in generale), che con i salariati (Balbi 1988). Garcia, sulla base della mancata attivazione degli investimenti privati, ricerca il confronto con $i$ gruppi attraverso il progetto, che poi fallirà, di nazionalizzazione del sistema bancario (separazione). La moratoria sul debito estero viene criticata, ma solo con riferimento allo stile negoziale ( $\mathrm{Fi}$ geroa 1991). Garcia si rifiuta di effettuare un cambiamento e adotta misure di compromesso con i salariati - i tagli ai salari sono minori di quanto i vari governi vorrebbero - ma l'effetto finale è la loro penalizzazione, che dipende più dall'iper-inflazione che dall'aggiustamento. Il conflitto cristallizza: durante il 1988 gli scioperi generali sono quattro, ma sempre a bassa intensità. L'ennesima crisi economica è la diretta conseguenza di questi eventi.

Il cambiamento di Fujimori è provocato anch'esso da una crisi, ma non era stato annunciato nella campagna elettorale del 1990; anzi era stato prospettato il contrario. Fujimori favorisce la coalizione liberista e ristabilisce l'alleanza con il FMI: non vi è un accordo formale, ma la politica economica viene appoggiata. Viene raggiunto un accordo di riscadenzamento con i governi creditori, ma il rifinanziamento non si è materializzato anche a causa dell'auto-golpe di Fujimori dell'aprile del 1992. Le banche creditrici continuano ad essere penalizzate dalla moratoria, ma un accordo, con loro e con il FMI, è in vista nel 1993. I grandi gruppi risolvono il conflitto con le privatizzazioni e soprattutto con la reintroduzione del mercato delle terre in agricoltura per rafforzare i legami con i produttori. Il conflitto potenziale con i produttori della costa per l'effetto negativo della politica creditizia viene quindi risolto attraverso uno scambio con la revoca completa della riforma agraria di Velasco. Il dominio nei confronti dei contadini diventa completo (frammentazione): infatti per i singoli contadini vendere le proprie terre e migrare in città è più redditizio che produrre. I gremios, che si erano opposti alla revoca della riforma agraria, finiscono quindi per non rappresentare più i contadini; ma anche le nuove organizzazioni di base (rondas campesinas) non sono capaci di cristallizzare il conflitto (Monge 1989). Il capitale filo-protezionista è svantaggiato dall'apertura commerciale, dal calo dei sussidi alle esportazioni industriali e dalla sopravvalutazione: il conflitto cristallizza, ma il dominio non viene evitato. Deve ancora essere definito se parte delle imprese pubbliche saranno mante- 
nute: l'ipotesi è positiva per il settore minerario e negativa per i settori peschiero e finanziario. I salariati non recuperano le perdite degli anni precedenti e continuano ad essere penalizzati (dominio): il conflitto cristallizza, ma i due scioperi generali hanno ancora scarso successo.

In Venezuela, l'aumento dei prezzi del petrolio nel 1973, durante il periodo protezionista di Perez, aveva permesso di favorire tutti gli attori interni. Herrera Campins penalizza i salariati (compromesso), ma soprattutto i marginali. Un progetto di cambiamento (apertura commerciale), che avrebbe svantaggiato il capitale filo-protezionista, rientra dopo il nuovo boom petrolifero del 1979. Tale settore è favorito da politiche di scambio: tra controlli dei prezzi e protezionismo (per gli industriali che producono per il mercato interno), tra sussidi e sopravvalutazione (per gli esportatori industriali), tra crediti e apertura (o austerità e protezionismo) per i produttori agricoli per il mercato interno (Hernandez 1987). Analoghi scambi vengono effettuati a favore del capitale filo-liberista (ad es. il settore finanziario), minoritario in Venezuela, dove non esiste una produzione agricola per l'esportazione. Dopo la svalutazione del 1983 e con Lusinchi, per il capitale non cambia la configurazione del conflitto, ma continua la penalizzazione dei salariati (compromesso), le cui remunerazioni non aumentano neanche con il passaggio alla fase di aggiustamento espansivo nel 1986. Il sindacato, controllato dal partito di governo (Acción Democrática), non cristallizza il conflitto né in questa fase né in quella precedente di aggiustamento restrittivo (Lucena 1991). Il FMI appoggia indirettamente la politica economica di Lusinchi, ma non vi sono accordi formali. Durante questi anni, a parte un paio di moratorie ininfluenti sul capitale, il debito viene sempre pagato (Rodriguez 1988). Il conflitto sul servizio del debito privato invece cristallizza puntualmente (Salgado 1987), ogni volta cioè che viene dichiarata una svalutazione: esso verrà sempre risolto con il compromesso (concessione di un tasso di cambio privilegiato per il suo servizio). Dato che il contesto della politica economica è protezionista, $\mathrm{i}$ grandi gruppi vengono compensati dai crediti (Perez I), dalla fuga di capitali (Herrera Campins), dalle speculazioni legate al sistema di tasso di cambio differenziato fissato da Lusinchi (Gomez 1989). L'effetto congiunto di questi eventi è la crisi economica.

Il cambiamento di Perez (il cosiddetto gran viraje), che è il prodotto della crisi economica ereditata da Lusinchi, porta al 
brusco passaggio alla fase di aggiustamento strutturale, che favorisce la coalizione liberista. Il Plan Tinoco, nascosto in campagna elettorale, passerà alla storia per aver provocato la rivolta (con 300 morti) dei settori marginali, il 27 febrero del 1989. Sulla base dell'alleanza (stavolta formalizzata) con il FMI, le banche creditrici sono temporaneamente penalizzate attraverso una moratoria parziale sugli interessi, ma non c'è conflitto tra esse e i grandi gruppi. Il conflitto cristallizza, ma viene risolto (con il convincimento del governo) dopo il boom del prezzo del petrolio nel 1991. I gruppi vengono privilegiati dalle privatizzazioni e dalla maggiore apertura agli investimenti esteri; il settore finanziario (capitale filo-liberista) dalla liberalizzazione del tasso di interesse; inoltre le esportazioni agricole sono incoraggiate attraverso una politica (l'unica) di sussidi statali. C'è un processo di cambiamento delle regole del gioco con la segmentazione del settore industriale: $\mathrm{i}$ produttori per il mercato interno subiscono l'apertura commerciale e la liberalizzazione dei prezzi (dominio); ma gli esportatori ricevono uno scambio tra taglio ai sussidi e liberalizzazione del tasso di cambio. I produttori agricoli per il mercato interno subiscono contemporaneamente il taglio ai crediti e l'apertura commerciale (dominio). Il conflitto cristallizza in entrambi i casi, anche se l'intensità di quello tra governo e produttori agricoli è maggiore: nel 1991 c'è stata una dimostrazione a Caracas. Non tutte le imprese pubbliche vengono privatizzate: il petrolio resterà allo stato. I salariati sono ancora penalizzati - le perdite maggiori derivano dall'ajuste del 1989 attraverso la flessibilizzazione del mercato del lavoro (dominio): il sindacato convoca senza convinzione un paio di scioperi generali sull'onda delle rivolte popolari nel 1989 e delle proteste degli studenti nel 1991.

In sintesi, il paese che si dimostra maggiormente inclusivo di attori favoriti sembra essere il Venezuela, e l'ipotesi è quella di una maggiore disponibilità di risorse a causa del petrolio. Tali risorse però hanno incoraggiato anche la corruzione: è stata proprio l'assenza di una riforma istituzionale a provocare una crisi di legittimità del regime (vedi i due tentativi di golpe nel 1992). Quello maggiormente esclusivo (soprattutto a livello internazionale) è invece il Perú, la cui crisi economica e politica è testimoniata dall'auto-golpe di Fujimori dell'aprile del 1992, che ha portato all'elezione di un'Assemblea Costituente, la cui legittimità è stata contestata da molti partiti. In Argentina, dopo i vari tentativi di golpe nel periodo di Alfonsin - la cui 
causa principale erano i processi contro i militari - vi è senz'altro più stabilità democratica.

\section{Attori e coalizioni}

Completata la sintesi sull'evoluzione storica dei tre paesi, è opportuno adesso svolgere una panoramica comparata sui sei principali attori ${ }^{20}$. Su tali attori era già stata fatta una premessa all'inizio del paragrafo precedente, che portava ad evidenziare gli elementi di somiglianza tra i casi. Nelle pagine che seguono saranno evidenziate (ed elencate) le diversità rispetto alla suddetta premessa. Inoltre verrà fatto un tentativo di spiegazione degli esiti del conflitto, con riferimento al ruolo dei governi o dei singoli attori, che è significativo soprattutto per gli attori penalizzati, cioè i membri della coalizione protezionista.

Il processo di inclusione dei creditori tra gli attori favoriti è stato lungo. Il conflitto è cristallizzato quando i governi hanno dichiarato delle moratorie sugli interessi: nel Perú di Garcia in modo costante e volontario; in modo transitorio e tacito in tutti i tre i paesi nella fase di aggiustamento strutturale. La penalizzazione dei creditori da parte del Perú di Garcia, rispetto alle opzioni di compromesso operate dagli altri governi, rappresenta il primo elemento di diversità tra i tre paesi: i motivi di ciò sono riconducibili al progetto politico populista di Garcia. Nel caso di Grinspun non vi fu una vera e propria moratoria - gli interessi vennero pagati, seppur a fatica - ma solo una politica di confronto con il FMI. Una differenza non riferita ai paesi, ma cronologica, riguarda il rapporto tra banche e FMI. Nella fase di aggiustamento strutturale infatti, in seguito al lancio del piano Brady da parte degli Stati Uniti, il coordinamento tra FMI e banche è venuto meno e il conflitto è spesso cristallizzato. Il FMI ha dato il proprio assenso all'accumulazione di arretrati sugli interessi bancari e ha approvato programmi di aggiustamento prima dell'accordo con le banche, in Argentina e in Perú (tacitamente). Tale strategia era comunque soltanto di breve periodo: le banche

${ }^{20}$ Per le imprese pubbliche valgono le considerazioni fatte in precedenza. Tali imprese, non essendo dotate di una struttura rappresentativa distinta dallo stato, non hanno capacità autonoma di risposta alle politiche economiche dei governi, che hanno ritardato essi stessi (vedi le conclusioni) le privatizzazioni. Gli unici a cristallizzare il conflitto sono eventualmente $\mathrm{i}$ lavoratori delle imprese pubbliche, che di solito vengono classificati tra i salariati. 
si sono accordate sulla fine della moratoria sia con il Venezuela che con l'Argentina, mentre un accordo è in vista (nel 1993) con il Perú. La modalità di risoluzione di lungo periodo è stato dunque il convincimento coercitivo dei governi debitori.

I grandi gruppi economici hanno cristallizzato il conflitto soprattutto in Perú con Velasco e in Argentina con Perón. L'incompatibilità non era solo sulle relazioni capitale/lavoro, ma riguardava la definizione della politica economica, del modello di sviluppo e di un progetto di società in generale. Nel breve periodo il conflitto è stato risolto con il dominio ai danni dei gruppi, ma nel lungo periodo i suddetti governi sono caduti, e quindi sono stati ridotti all'impotenza. Negli anni ottanta il conflitto è di nuovo cristallizzato sia in Perú che in Argentina. Garcia, in rappresaglia contro i mancati investimenti privati, decise la nazionalizzazione del sistema bancario, ma il governo non riuscì ad attuare tale progetto e il conflitto fu risolto con la separazione. In Argentina, sia Alfonsin (nell'ultima fase) che, soprattutto, Menem (nella prima fase) hanno tentato di limitare il potere dei gruppi del polo contratista, ma alla fine è stato applicato il compromesso: i conflitti comunque sono cristallizzati, ma con Cavallo gli indicatori economici sono migliorati e il conflitto con i gruppi è rientrato. Va sottolineato che la patria contratista fa parte della coalizione protezionista e quindi osteggia l'aggiustamento strutturale sulla situazione argentina influì inoltre l'intensità del conflitto distributivo che portò al confronto tra gruppi e creditori.

Il tentativo di penalizzazione dei gruppi da parte di Garcia rappresenta un secondo elemento di diversità tra $i$ tre casi: le ragioni di ciò sono le stesse del conflitto con i creditori. In Venezuela, come detto, c'è una maggiore integrazione tra grandi gruppi e capitale estero: i gruppi sono stati favoriti anche in periodi di protezionismo, attraverso l'incoraggiamento della fuga di capitali e degli investimenti all'estero (diversione). Nella fase di aggiustamento incompleto infatti, la fuga di capitali è servita ai gruppi come strumento principale di diversione, in assenza delle riforme strutturali. Misure come le privatizzazioni, l'apertura agli investimenti esteri in campo produttivo e finanziario vengono attuate solo nella fase attuale di aggiustamento strutturale. Nel lungo periodo dunque il conflitto con i grandi gruppi è stato risolto con il convincimento coercitivo ai danni dei governi, che in passato avevano ritenuto che la lotta contro di essi fosse lo strumento principale per innescare un equo processo di sviluppo. I gruppi ricevo- 
no molti benefici, ma si rifiutano di fare concessioni: la riforma tributaria è ancora il nodo principale che impedisce l'equità ${ }^{21}$.

Per ciò che riguarda il capitale filo-liberista, in periodi protezionisti il conflitto tra governo e produttori agricoli per l'esportazione è stato risolto con lo scambio. Alcuni conflitti cristallizzati, la cui modalità di risoluzione iniziale era il dominio (come nei casi di Perón, Grinspun e del Plan Primavera in Argentina), sono stati risolti nel lungo periodo con la riduzione all'impotenza dei governi. In Perú non è cristallizzato né il conflitto sulla riforma agraria - l'associazione dei latifondisti fu ridotta all'impotenza - né quello successivo sulla politica creditizia restrittiva. Gli imprenditori rurali infatti hanno ottenuto come compensazione la progressiva sconfitta delle conquiste delle riforma: la parcellizzazione delle cooperative con Belaunde, la reintroduzione del libero mercato delle terre con Fujimori. Lo scambio è stata la modalità prevalente di risoluzione del conflitto anche per gli altri settori del capitale filo-liberista, sempre in periodi protezionisti. Gli esportatori tradizionali (settori minerario e della pesca) in Perú hanno ricevuto crediti, mentre per le banche $^{22}$ c'è stata una sorta di divisione del lavoro ${ }^{23}$. Il conflitto è cristallizzato solo con Velasco a causa delle nazionalizzazioni ai danni dei settori minerario, peschiero e finanziario. La stessa configurazione del conflitto si era avuta in Argentina con Perón. Il dominio era stato imposto verso tutti questi settori, ma i governi sono stati convinti alla necessità di riprivatizzare solo nella fase attuale di aggiustamento strutturale - per il settore minerario, ciò non è ancora sicuro. In questa fase dunque, il capitale filo-liberista rientra tra gli attori favoriti, senza eccezioni.

${ }^{21}$ Le riforme attuali mirano a perfezionare il sistema dell'IVA - tassa che pagano i consumatori - $\mathrm{e}$ ad abbassare $\mathrm{i}$ tassi nominali impositivi, esonerando $\mathrm{i}$ contribuenti più poveri. Mentre la riforma delle imposte dirette incontra consenso - tutti ne sono beneficiati -, quella dell'IVA ha l'opposizione dei salariati.

${ }_{22}$ Le banche hanno accettato controlli al tasso di interesse, dato che il settore finanziario pubblico si accollava i maggiori rischi creditizi, con prestiti di medio e lungo periodo. L'eccezione è l'Argentina, dove, a partire dal 1977, i tassi sono stati liberalizzati: la suddetta divisione del lavoro non si è consolidata, anche per il fattore disturbo rappresentato dal settore delle banche estere, che sono restate in Argentina, al contrario degli altri paesi.

${ }^{23}$ La divisione del lavoro (scambio) è stata applicata anche agli importatori. Essi si sono specializzati nelle importazioni di beni intermedi e capitali, dato che i beni di consumo sono stati sempre protetti per favorire l'industria locale. L'eccezione in questo caso è rappresentata dal Perú, dove, a partire dal 1970, la maggior parte degli importatori si sono riconvertiti in industriali, e, in periodi di apertura, sono stati gli stessi produttori a importare. 
A proposito del capitale filo-protezionista, nella fase di aggiustamento strutturale, il conflitto tra governo e produttori industriali per il mercato interno sull'apertura commerciale è stato risolto con il dominio. I conflitti sono cristallizzati, ma con più intensità da parte delle associazioni di secondo grado (cioè dei singoli settori industriali), che di quelle di terzo grado (le varie confindustrie). Per non essere isolati dagli altri settori imprenditoriali, tali associazioni, pur obiettando all'apertura, hanno accettato le riforme strutturali: è stato questo fattore ideologico che le ha (in parte) frenate. Il conflitto era cristallizzato (in Perú) oppure era stato risolto in dominio (in Argentina) anche nei periodi liberisti alla fine degli anni settanta, ma le politiche di apertura erano state poi ritirate (scambio). Lo scambio è stato la modalità prevalente di risoluzione del conflitto anche nella fase di aggiustamento incompleto (anni ottanta), quando i conflitti non sono cristallizzati. Il conflitto riguardante gli esportato$r i$ industriali è stato sempre risolto dallo scambio, che in passato penalizzava attraverso la sopravvalutazione, attualmente attraverso il taglio ai sussidi. Si può quindi affermare che per questo settore, l'effetto dell'aggiustamento sia neutrale, nel senso che viene delegato alla competitività dei singoli produttori. Il contemporaneo ricorso alla sopravvalutazione in Argentina e Perú trasforma lo scambio in dominio e rappresenta un terzo elemento di diversità tra i paesi, i cui motivi vanno identificati nelle scelte di politica economica di Cavallo in Argentina - che però alla fine del 1992 ha reintrodotto alcuni sussidi (compromesso) -, e nel peso dei narco-dollari - che creano un mercato cambiario parallelo in Perú. In passato, c'erano state discriminazioni nel settore industriale ${ }^{24}$ con Morales Bermudez (a favore degli esportatori) e durante il governo Grinspun (a favore dei produttori per il mercato interno). I produttori agricoli per il mercato interno in Venezuela hanno subito il dominio nella fase di aggiustamento strutturale: il conflitto è cristallizzato sia a livello di terzo che di secondo grado, ma per i produttori, piuttosto deboli perché poco numerosi, resta l'opzione della riconver-

${ }^{24}$ La piccola industria ha subito il dominio in periodi liberisti (Martinez de Hoz, Belaunde II, ma non Morales Bermudez): il conflitto è cristallizzato, ma le associazioni rappresentative del settore sono così deboli, che la sua intensità resta a livelli molto bassi. Nella fase attuale di aggiustamento strutturale non sempre il conflitto cristallizza ma il dominio non viene evitato - a causa delle illusioni sugli effetti anti-concentrazionisti del liberismo (in Venezuela) o sulle potenzialità esportatrici del settore (in Perú). 
sione verso le esportazioni. Il capitale filo-protezionista rientra dunque tra gli attori penalizzati dall'aggiustamento strutturale. L'exit dei singoli imprenditori è individuale (frammentazione): importare, speculare in finanza, oltre che (naturalmente) chiudere. C'è segmentazione solo se le esportazioni, industriali o agricole, sono favorite.

I salariati rientrano tra gli attori penalizzati dall'aggiustamento strutturale, attraverso l'instaurazione di un rapporto di dominio $^{25}$. La mancata cristallizzazione nel caso della CTV (Confederación Trabajadores Venezolanos) in Venezuela, a parte una reazione strumentale dopo le rivolte del 27 febrero del 1989 e le proteste degli studenti del 1991, è imputabile alla scarsa propensione al conflitto di leaders sindacali premiati in passato dalla rendita petrolifera e dediti attualmente al mantenimento dei propri privilegi. In Perú, dove la CGTP (Confederación $\mathrm{Ge}$ neral Trabajadores Peruanos) è indipendente dai partiti al potere, il conflitto è cristallizzato in concomitanza con l'aggiustamento, ma le proteste non hanno avuto successo. In questo paese ha senz'altro influito la memoria del fallimento delle strategie conflittuali degli anni settanta, dove l'alleanza con i lavoratori del settore informale non aveva impedito la repressione della leadership sindacale. Nella fase degli esperimenti neo-liberali alla fine degli anni settanta, i regimi militari avevano imposto il dominio sia in Argentina che in Perú, ma solo in quest'ultimo caso il conflitto era cristallizzato, perché minore era stata la repressione: molti desaparecidos argentini erano sindacalisti della CGT (Confederación General de Trabajo). Negli anni ottanta, la modalità prevalente di risoluzione del conflitto tra governo e sindacati era stata il compromesso (e talvolta lo scambio). La cristallizzazione del conflitto nel caso della CGT in Argentina è dipesa da variabili politiche - il partito radicale era al potere e il peronismo all'opposizione - e in parte dalla leadership carismatica e conflittuale di Ubaldini. Con Menem, grazie

${ }^{25}$ Questo giudizio è limitato naturalmente agli effetti di breve periodo dell'aggiustamento. Secondo gli studiosi di orientamento liberista, tali effetti sono stati causati più dall'iper-inflazione e saranno più che compensati nel medio e lungo periodo dalla crescita economica, favorita dalle riforme strutturali - il caso di riferimento è ancora una volta quello cileno. Il compito di un politologo è quello di verificare tali ipotesi: per ciò che riguarda il passato, l'enfasi sull'inflazione è giustificata sia in Argentina che in Perú, ma non c'è caso che ha accoppiato aggiustamento e crescita salariale; per ciò che riguarda il futuro, almeno fino alla fine del 1992 non vi è stata, in questi tre paesi, alcuna ripresa salariale. 
alle segmentazione che ha penalizzato Ubaldini, il conflitto non è cristallizzato proprio per lo stesso motivo, cioè la mancanza di autonomia della CGT dal partito peronista - lo sciopero del novembre del 1992 non rappresenta una significativa inversione di tendenza. L'andamento dei salari, il cui calo è stato il risultato sia dell'iper-inflazione (in Argentina e in Perú) che di un forte shock (in Venezuela), ha rappresentato il terreno principale di incompatibilità. Nella fase di aggiustamento strutturale, si è aggiunto un altro obiettivo incompatibile: la flessibilizzazione del mercato del lavoro (riduzione della stabilità del lavoro, minor potere dei negoziati collettivi, limitazione del diritto di sciopero), soprattutto nel contesto delle privatizzazioni che, almeno in una fase iniziale, comportano licenziamenti. In questa fase, l'opposizione (senza successo) è venuta più dai singoli settori di salariati (soprattutto gli impiegati statali) che dalle centrali sindacali. In sintesi, intensità e cristallizzazione del conflitto hanno coinciso solo in Argentina durante la presidenza Alfonsin: la resistenza dei sindacati in questo caso rappresenta un quarto elemento di diversità tra $\mathrm{i}$ tre paesi. In Perú invece $\mathrm{i}$ salari non sono diminuiti per un'opzione politica di Garcia: quando nel 1988 il governo li tagliò, la CGTP non fu capace di opporsi. Oltre alle spiegazioni già citate per i singoli casi, facenti riferimento a variabili quali l'autonomia dai partiti, la leadership, il meccanismo di cooptazione dei leaders sindacali, ve ne sono altre, che invece sono rintracciabili in tutti e tre $\mathrm{i}$ casi. Si tratta di due tipi di spiegazioni: da un lato quelle più tradizionali riferite alla segmentazione attraverso il premio salariale a determinate categorie (i metallurgici in Argentina, i petroliferi in Venezuela, i minatori in Perú) e alle tendenze macroeconomiche che favoriscono la de-industrializzazione; dall'altro lato quelle, a mio avviso più valide, che afferiscono sia a variabili individuali - per effetto della crisi, i lavoratori non partecipano agli scioperi politici (e in parte neanche a quelli settoriali), per timore di essere licenziati - che collettive - un certo consenso da parte dell'opinione pubblica nei confronti delle riforme strutturali e quindi il timore che gli scioperi possano essere condannati perché corporativi. Così, non scioperando per paura dei licenziamenti, l'exit dei salariati è quello individuale (frammentazione): la ricerca di un secondo lavoro e l'uscita verso il settore informale.

Gli esclusi urbant ${ }^{26}$ sono stati protagonisti dei cosiddetti

${ }^{26}$ Per ciò che riguarda gli esclusi rurali, le conclusioni riguardano solo il Perú: ne- 
estallidos (esplosioni) sociales che si sono verificati in tutti e tre i paesi: Venezuela (febbraio 1989); Argentina (maggio 1989) e Perú (luglio 1990). In Argentina, le rivolte sono state il risultato del processo iper-inflazionario; negli altri due paesi le esplosioni si sono verificate in seguito all'aumento dei prezzi dei beni di prima necessità per la contemporanea riduzione dei sussidi statali. È azzardato intravedere in queste rivolte l'inizio di una rivoluzione dei marginali, come hanno fatto gli studiosi terzomondisti. Un'ipotesi più coerente è che gli estallidos si siano verificati laddove i marginali non fossero organizzati. Le proteste infatti sono state deboli in Perú dove vi sono molti grupos de vecinos, e più violente negli altri due paesi dove tali settori sono meno organizzati ${ }^{27}$. La diversità nell'intensità degli estallidos rappresenta il quinto elemento di diversità tra i tre paesi. Il bilancio finale è quello di una forbice tra esplosioni disorganizzate (in Argentina e Venezuela) ed implosione organizzata (in Perú) che non risolve il rapporto di dominio. L'aumento dell'organizzazione dei marginali (indicatore del livello comportamentale del conflitto) è ostacolato dall'aumento della delinquenza (indicatore del livello attitudinale), osservato in Perú e in Venezuela. Sendero Luminoso ${ }^{28}$ sta tentando di legare l'implosione urbana (violenta) con l'organizzazione della violenza (rurale), reclutando i giovani marginali (delinquenti o meno) di Lima o gli universitari radicalizzati delle città della sierra.

In sintesi, gli elementi di somiglianza che sono spiegati sulla base di variabili internazionali (vedi l'evoluzione storica all'inizio del paragrafo sui casi), prevalgono su quelli di diversità. Le spiegazioni di questi ultimi fanno riferimento in parte alle scelte dei governi (il populismo di Garcia nel conflitto con i creditori e i grandi gruppi), in parte alle caratteristiche degli attori politici (la CGT in Argentina) o dei gruppi sociali (il settore marginale/informale in Perú). Il caso degli esportatori in-

gli altri due paesi i contadini sono pochi e per motivi di spazio questi casi non sono stati trattati. Dato il basso livello dei paros dei contadini, si può ipotizzare che anche in contesto rurale prevalga l'implosione, che però non si traduce in violenza diretta, $\mathrm{ma}$ in un sentimento di sfiducia nello stato. Un motivo di fallimento della politica creditizia di Garcia è stato il fatto che i contadini utilizzavano il denaro per il contrabbando.

${ }_{27}$ Per ciò che riguarda altre possibili spiegazioni di questi eventi, rimando a Fossati (1993).

${ }^{28}$ Sendero non è stato analizzato come attore perché le sue posizioni sono troppo radicali per stabilire un legame con il debito o l'aggiustamento. Comunque, sembra che finora i pobladores organizzati e le comunità contadine resistano agli attentati terroristici compiuti da Sendero nei loro confronti. 
dustriali è più controverso: l'effetto neutrale dell'aggiustamento strutturale è confermato solo in Venezuela, ma è forse più corretto affermare che l'esito di questo conflitto derivi da fattori contingenti.

\section{Conclusioni}

Il bilancio della fase attuale di aggiustamento strutturale corrisponde dunque alla vittoria della coalizione liberista su quella protezionista; se tale fenomeno si consoliderà nei prossimi anni, si potrà parlare di coalizione egemonica - termine che è appropriato per la coalizione protezionista, solo con riferimento agli anni cinquanta. I governi che hanno tentato di colpire i grandi gruppi, o soltanto il capitale filo-liberista, in misura minore (Grinspun, Sourrouille con il Plan Primavera), o maggiore (Perón, Velasco e Garcia) prima o poi sono stati ridotti all'impotenza. Nelle pagine seguenti, ripercorrerò il processo di destrutturazione progressiva del modello protezionista, anticipato nel paragrafo sui casi, alla ricerca di spiegazioni, ma senza la pretesa di dare una risposta definitiva.

Fin da quando sono state poste le fondamenta del modello dell'industrializzazione per la sostituzione delle importazioni, attraverso le nazionalizzazioni e i vari strumenti di politica economica, i governi hanno modificato la struttura di potere esistente tra i diversi attori. Quindi, ricorrendo al modello di Emerson (1962), che definisce le tattiche per rompere una relazione di dipendenza tra due attori con un accesso asimmetrico alle risorse, si può sostenere che i governi hanno fatto ricorso alla quarta tattica, cioè la modifica dell'accesso alle risorse della coalizione liberista per favorire la coalizione protezionista. Ciò è potuto avvenire quindi solo attraverso il processo autoritativo di allocazione delle risorse, compito che è demandato allo stato che detiene il monopolio dell'uso legittimo della forza. Questo modello comunque non prevede, come il socialismo, la riduzione all'impotenza del capitale privato attraverso la sua soppressione, ma solo il tentativo di instaurare un rapporto di dominio nei confronti del capitale filo-liberista e dei grandi gruppi, che infatti sono stati solo limitati nella dotazione delle proprie risorse. Questi due attori hanno reagito non solo con il calo degli investimenti e la fuga dei capitali, ma soprattutto con l'evasione fiscale. Privati di questo strumento, i governi hanno dovuto so- 
stenere l'espansione monetaria con il deficit pubblico e l'inflazione. La crisi economica e la polarizzazione politica sono state risolte in taluni casi solo con l'intervento dei militari.

In ogni caso, le reazioni dei suddetti attori non hanno pagato subito: nella fase degli esperimenti neo-liberali alla fine degli anni settanta l'aggiustamento non ebbe successo, soprattutto perché non vi furono privatizzazioni, a parte il caso del Cile. Le privatizzazioni infatti comportano la messa in discussione di molti interessi consolidati per ciò che riguarda sia i dirigenti, che i salariati. In ogni caso, una spiegazione di tipo «italiana», cioè che $\mathrm{i}$ governi non privatizzano se guidati dai membri di quei partiti legati alle imprese pubbliche, convince solo in parte. In casi come l'Argentina, c'erano i militari al potere: vi fu un'opposizione di carattere nazionalista delle forze armate proprio verso la politica di privatizzazioni, e non verso l'aggiustamento monetario e salariale. Nel caso del Perú (prima e dopo la transizione), contò anche l'opposizione dei salariati: inoltre sarebbe stato difficile far rientrare tutte le riforme di Velasco, specialmente le nazionalizzazioni che avevano avuto un alto consenso popolare. In Venezuela, il petrolio permise il rinvio della crisi e di molte politiche impopolari, soprattutto durante la presidenza di Herrera Campins, che era di orientamento più liberista.

Con il ritorno alla democrazia, il modello protezionista riprese ad essere applicato, ma in contemporanea all'aggiustamento. Fu decisiva la pressione dei creditori esteri, che comunque non bastò a far applicare le riforme strutturali (né apertura commerciale, né privatizzazioni). I governi erano tutti controllati dai partiti, e, per ciò che riguarda i lavoratori, c'era già la difficoltà di far accettare i tagli salariali ${ }^{29}$. L'aggiustamento, come nella fase precedente, non ebbe successo, e la crisi economica fu aggravata dalle reazioni dei membri della coalizione liberista già specificate in precedenza, rafforzati dai creditori, non solo attraverso la costrizione del debito, ma anche con la speculazione cambiaria. Con l'intensificarsi del conflitto distributivo, anche il settore industriale si mobilitò attraverso gli aumenti dei prezzi, che, in un regime protezionista, sono concordati dai maggiori produttori. Tale settore inoltre non fu capace di con-

${ }^{29}$ Con la trasposizione sui prezzi degli aumenti salariali, gli industriali scaricano i costi della crisi sui salariati: l'unico modo di vincere l'inflazione diventa quindi la deindicizzazione salariale. 
solidare solide alleanze all'estero: vi furono sì investimenti di imprese straniere, ma gli spin-offs legati al modello protezionista, soprattutto per le scarse capacità esportatrici di un'industria inefficiente, erano limitati.

Il risultato finale fu l'ennesimo fallimento del modello protezionista che portò alla sua condanna pressoché definitiva da parte dell'opinione pubblica. Per la prima volta, i governi democratici della fine degli anni ottanta sono stati messi nelle condizioni di applicare le riforme strutturali, spezzando il circolo vizioso del meccanismo delle reazioni previste. Questo risultato è stato però raggiunto solo dopo aver toccato il fondo, con l'iper-inflazione. Il modello protezionista, in passato, aveva rappresentato la maggiore speranza politica, al fine di rompere la dipendenza della «periferia» dal «centro». La precedente sconfitta (economica e non solo politica) dei governi che ne avevano rappresentato la massima espressione (nei primi anni settanta con Allende e il ritorno di Perón) era stata quasi dimenticata con il ritorno alla democrazia, spesso vissuta con troppe illusioni (vedi i casi di Alfonsin e di Garcia): l'opinione pubblica non avrebbe ammesso le riforme strutturali. Nella fase attuale invece, l'unica speranze per i membri della coalizione protezionista è l'exit individuale (frammentazione). Il tipo di relazione tra stato e capitale filo-protezionista (le imprese pubbliche e il settore industriale inefficiente), basato sulla protezione dall'esterno e sulla sottrazione dalle regole di mercato, è ormai delegittimato. I salariati sperano che i sacrifici derivanti dall'aggiustamento comportino un futuro migliore grazie alla crescita economica attivata dalle riforme strutturali; inoltre in questa fase si configurano nuove identità collettive, come quella dei consumatori, che vengono favoriti sia dal calo dell'inflazione che dall'apertura commerciale ${ }^{30}$.

L'articolo a questo punto avrebbe già trovato le sue conclusioni, ma la problematica della relazione tra livello internazionale e livello interno non può essere, a mio avviso, lasciata da parte. Intendo dunque ritornare sull'ipotesi di ricerca, riguardante

${ }^{30}$ Chi ha risorse, organizzazione ma scarsa capacità di mobilitazione (il capitale filo-protezionista), non è riuscito ad ottenere la conformità degli altri attori, al contrario del capitale filo-liberista che si è coalizzato con $\mathrm{i}$ grandi gruppi. Chi ha poche risorse (i salariati) deve organizzarsi, ma ciò non è bastato ad assicurare la mobilitazione della base. Infine, chi non ha risorse (gli esclusi), può al massimo aspirare a mobilitarsi, ma solo nel breve periodo, attraverso gli estallidos sociales, non essendo capace di trasformare la mobilitazione in organizzazione. 
la relazione inversa tra conflitto sul debito e sull'aggiustamento, seppur corretta dal filtro delle coalizioni. Tale ipotesi, che dà luogo a due possibilità, può essere esposta nel modo seguente: se il governo debitore dichiara una moratoria, privilegiando le relazioni con le imprese pubbliche, il capitale filo-protezionista e i salariati, il conflitto cristallizza con la coalizione liberista; se c'è un accordo tra governo, creditori, grandi gruppi e capitale filo-liberista per pagare il debito, il conflitto cristallizza con la coalizione protezionista. Le due possibilità possono essere presentate anche attraverso due modelli: quello Nord-Sud - il paese debitore fa prevalere gli interessi dei paesi in via di sviluppo, dichiarando una moratoria - e quello centro-periferia - il paese debitore privilegia $\mathrm{i}$ creditori, sulla base della supposta alleanza tra i topdogs (Cardoso e Faletto 1979).

L'analisi empirica mostra che il modello che accoppia il servizio del debito al conflitto tra governo e coalizione protezionista non solo ha avuto più riscontri empirici, ma ha alla fine prevalso. In realtà tale modello non è stato rispettato a pieno, per una sorta di paradosso «scientifico» tra le due variabili principali della teoria del conflitto: cristallizzazione e risoluzione. Infatti, i tre attori della coalizione protezionista subiscono il dominio, ma solo le associazioni imprenditoriali del capitale filoprotezionista (e non i salariati) hanno cristallizzato, seppur senza successo, il conflitto - le imprese pubbliche invece non possono strutturalmente farlo. I motivi di tutto ciò sono stati approfonditi nelle pagine precedenti.

Il modello Nord-Sud, che accoppia la moratoria al conflitto tra governo e coalizione liberista (grandi gruppi e capitale filoliberista), è stato smentito dai fatti. La mancata attivazione dei grandi gruppi in Perú contro la moratoria di Garcia è dovuta al fatto che i crediti esteri ai privati negli anni settanta, e di conseguenza anche la fuga di capitali, sono stati di entità minore rispetto agli altri paesi: il legame tra creditori e gruppi è stato più debole. La cristallizzazione successiva dipese inoltre dal confronto ricercato da Garcia contro i grandi gruppi attraverso le ben note misure del luglio del 1987 e non dalla moratoria. Alla base del calo degli investimenti dei gruppi vi fu il fallimento del cosiddetto programma eterodosso che $i$ gruppi avevano, se non appoggiato, tollerato. Il caso di Grinspun conferma la cristallizzazione del conflitto (sulla politica economica) con la coalizione liberista, ma è falsato dal fatto che non vi fu una vera e propria moratoria - gli interessi vennero pagati, seppur a fatica -, ma 
solo una politica di confronto con il FMI. Negli altri tre casi di moratoria, relativi alla fase di aggiustamento strutturale, è l'ipotesi stessa a subire una modifica fondamentale: la contemporanea presenza di moratoria e aggiustamento infatti fa saltare le ipotesi sui conflitti degli attori interni. Il motivo del silenzio dei grandi gruppi venezuelani e argentini (del polo liberista) è l'atteggiamento positivo verso chi applica l'aggiustamento strutturale. Le moratorie inoltre erano transitorie, tacite e appoggiate dal FMI. La patria contratista argentina, più vicina alla coalizione protezionista, si è mobilitata invece in favore della moratoria, cristallizzando il conflitto con i creditori, quindi come prevedeva l'ipotesi. Infine, il capitale filo-liberista, proprio perché meno legato ai creditori, non si è mobilitato contro la moratoria in nessuno dei tre casi.

La relazione inversa prevista dall'ipotesi ${ }^{31}$ è stata smentita dunque sia nell'unico caso di moratoria duratura (il Perú di Garcia), per ciò che riguarda la coalizione liberista, che nella maggioranza delle situazioni che presentavano l'accoppiata più comune tra servizio del debito e aggiustamento, per ciò che riguarda la coalizione protezionista. Un caso anomalo, non previsto dai modelli ma che rappresenta una parziale conferma dell'ipotesi, si è avuto quando ad una moratoria transitoria si è accoppiato l'aggiustamento strutturale, e il polo contratista (e filoprotezionista) dei grandi gruppi argentini si è schierato contro i creditori.

\section{Riferimenti bibliografici}

Acuña, C.H. (1990), Intereses empresarios, dictadura y democracia en la Argentina actual, CEDES, Buenos Aires.

Balbi, C.R. (1988), Las relaciones estado-sindicalismo en el Peru (19851987), FFE, Lima.

Ballon, E. (a cura di) (1990), Movimientos sociales: elementos para una relectura, DESCO, Lima.

Cardoso, F.H. e E. Faletto (1979), Dependency and Development in Latin America, University of California Press, Berkeley.

Durand, F. (1987), Los empresarios y la concertación, FFE, Lima.

${ }^{31}$ Non ho ritenuto opportuno elaborare altri modelli, proprio perché ritengo che questi debbano seguire il principio della precisione analitica e non quello dell'indispensabile aderenza empirica. Inoltre, la realtà empirica non fornisce le premesse neanche per disegnare una seconda ipotesi di relazione tra livello interno e internazionale. 
Emerson, R.M. (1962), Power-dependence relations, in «American Sociological Review», XXVII, pp. 31-41.

Figeroa, L. (1991), Crisis and moratorium in Peru, articolo presentato al XXV Congresso Mondiale dell'IPSA, Buenos Aires, 21-25 luglio 1991.

Fossati, F. (1991), Debito estero e aggiustamento economico in America latina, in «La Comunità Internazionale», XLVI, pp. 556-577.

- (1993), Debito estero ed estallidos sociales in America latina, in «Relazioni Internazionali», VI, n. 22 (di prossima pubblicazione).

Galtung, J. (1989), Solving conflicts: a Peace Research Perspective, working paper, University of Hawaii Institute for Peace, Honolulu.

Gaudio, R. e A. Thompson (1990), Sindicalismo peronista, gobierno radical: los años de Alfonsin, FFE/Folios Editores, Buenos Aires.

Gomez, E. (1989), El empresariado venezolano: a mitad de camino entre Keynes y Hayek, CEDICE, Caracas.

Hernandez, J.L. (1987), Estado y politica agrícola, COPRE, Caracas.

Lucena, H. (1991), Las relaciones de trabajo: desafios y propuestas, Editorial Nueva Sociedad, Caracas.

Machinea, J.L. e J.F. Sommer (1990), El manejo de la deuda externa en condiciones de crisis de balanza de pagos: la moratoria argentina de 1988-89, Documentos CEDES (n. 59), Buenos Aires.

Monge, C. (1989), Las demandas de los gremios campesinos en los 80, «Debate Agrario», II, pp. 41-60.

Nun, J. e M. Lattuada (1991), El gobierno de Alfonsin y las corporaciones agrarias, Manantial, Buenos Aires.

Ostiguy, P. (1989), Los capitanes de la industria, Editorial Legasa, Buenos Aires.

Rodriguez, M.M. (1988), Venezuela y la negociación de le deuda externa: experiencia reciente y perspectivas, in $\mathrm{R}$. Bouzas, Entre la heterodoxía y el ajuste, Grupo Editorial Latinoamericano, Buenos Aires, pp. 273-298.

Salgado, R. (1987), Economic pressure groups and policy making in Venezuela: the case of Fedecamaras, in «Latin American Research Review», XXII, pp. 91-106. 